\title{
A comparative study on knowledge, attitude, and practice of injection safety among nurses in two hospitals in Ibadan, Nigeria
}

\author{
Adejumo P.O. ${ }^{1}$, Dada F.A. ${ }^{2}$
}

1. Department of Nursing, Faculty of Clinical Sciences, College of Medicine, University of Ibadan, Ibadan 2. Accident and Emergency Unit, Taraba State Specialist Hospital, Jilango

doi: 10.3396/ijic.v9i1.004.13

\begin{abstract}
This descriptive study took place in University College Hospital and Adeoyo Maternity Teaching Hospital, Ibadan. Convenience Sampling Technique was used for selecting study participants while data collection was done with the aid of questionnaire. Following ethical approval, a total of 295 and 105 questionnaires were administered face to face respectively in the two participating centers. However, only 385 were fit for analysis. The mean age of the respondents was 37 years and most of them (92.5\%) were females. All have heard about injection safety. Their knowledge level was high, $70.4 \%$ knew that unsafe injection predisposes to blood-borne infection, $55.9 \%$ had correct information that two-handed recapping is not a safe injection practice while, $84.4 \%$ claimed that contaminated sharps predisposes the community to bio-hazards and 293 (76.1\%) had correct information that used syringes and needles should be discarded in a sharp waste box. However, the high knowledge was not translated to practice. Half (50.4\%) of the recently sustained injuries was through intramuscular or subcutaneous injections. Only $15.6 \%$ reported the injuries to their institution. Out of the total respondents, $62.9 \%$ knew that their hospitals have injection policies while $53.2 \%$ said that nurses are not involved of such policies. Doctors were alleged by $79.5 \%$ as the health care workers who most frequently leave sharps at the patients' bed side. Nurses, as the nerve centre of the healthcare enterprise, must be advocates of safe injection practices.
\end{abstract}

\section{Key words}

Nurse's practice patterns; Knowledge; Attitude of health personnel; Nurses; Syringes and microbiology; Needles and microbiology

\section{Corresponding Author}

P. O. Adejumo

Department of Nursing, Faculty of Clinical Sciences,

College of Medicine, University of Ibadan, Ibadan, Nigeria

Tel. +234 8033923260

E-mail: bisiandbayo@yahoo.com 


\section{Introduction}

Medical treatment is intended to save life and improve health, and all health workers, especially nurses as the most populated healthcare workers who spend the most time with the patients, have a responsibility to prevent transmission of health-care associated infections. Adherence to safe injection practices and related infection control is part of that responsibility as it protects patients and health workers. The purpose of this study is to explore the knowledge, attitudes and practices of injection safety among nurses in two selected hospitals in Ibadan. The following are the specific objectives: (a) to assess nurses' knowledge on injection safety, (b) explore their attitudes towards injection safety, (c) find out if health facilities meet necessary injection safety requirements, and (d) identify unsafe injection practices that may be targeted for injection safety intervention activities. The study therefore seeks to provide answers to the following questions: What is the level of knowledge of nurses about injection safety? What is their attitude towards injection safety practices? What are the unsafe injection practices to be targeted for injection safety interventions? Do the selected hospitals have the necessary requirements for ideal injection safety? Are there hospital policies about injection safety in selected setting and what is the level of involvement of nurses in such policies?

\section{Background}

Individuals seek health care for preventive, curative or rehabilitative reasons. Diverse measures used in meeting the health needs of clients include nursing care, counselling, health information and health education, dietary care, physiotherapy, and pharmacological care. The pharmacological aspect include injectables which may pass through diverse routes like intramuscular, intravenous, intrathecal, intracardiac, intradermal, and subcutaneous. Although, these routes have their respective side effects and complications, intramuscular injections appear to be the most implicated in the transfer of infections according to Canadian Needle Stick Surveillance Network (CNSSN). ${ }^{1}$ Injections are the most common health care procedure worldwide and $70 \%$ of them are said to be unnecessary and oral medications could have worked in some cases where injections were prescribed and administered. ${ }^{2}$ Injections should therefore be administered safely when they are medically indicated; to avoid placing patients at risk of morbidity and mortality. Hutin and colleagues asserted that the best control practices for infections is the use of a new, single use injection device for each injection and for reconstitution of each unit of medication. ${ }^{3}$ These single use injection devices are widely available at cost effective prices, but according to Dicko and colleagues single use injection devices must be systematically funded by the health care institution, as failure to do so is a key determinant to widespread reuse of syringes and needles in the absence of sterilization in immunization services. ${ }^{4}$ Moreover, Logez stated that interventions to increase the availability of injection devices in curative services have improved injection safety. ${ }^{5}$

\section{Methodology}

The study was carried out in University College Hospital (UCH) and Adeoyo Maternity Hospital, Ibadan (AMTH). It covered Medical, Surgical, Paediatrics, Accident and Emergency, Obstetrics and Gynaecology units of the two hospitals.

Convenience sampling technique was used to select nurses who frequently come in contact with patients' body fluid. Out of the total population of 936 nurses in $\mathrm{UCH}, 282$ who were available at the time of data collection and willing to participate in the study were selected, while the total population of nurses in AMTH was 210 out of which 84 who were willing to sign consent form and available during data collection were selected. The total number of participants then was 366 and calculated with the attrition figure of 34 to make 400 . However only 385 questionnaires were retrieved and found fit for analysis.

A structured questionnaire divided into 4 parts was used to gather information as follows:

Section A: is an 8-item question about demographic characteristics of the respondents.

Section B: is a 13-item question exploring their knowledge about injection safety.

Section C: is a 20-item, five point Likert scale question on the nurses' attitude.

Section D: consists of 27 questions that explored the respondents' practices on injection safety.

Face and content validity were ascertained by expert opinions and suggestions of other researchers 
on injection safety, nurse clinicians as well as the statistical analyst, while test retest for the consistency, stability and accuracy of the instrument was done and it yielded a reliability correlation coefficient of 0.77 for questions on knowledge of injection safety, 0.81 for questions on attitude and 0.91 for practice of injection safety.

The researchers and the research assistants distributed the questionnaires through face to face approach following adequate explanation on the purpose of the study to participants and obtaining their written consent. Respondents' questions were answered and they were instructed on how to fill the questionnaires which were retrieved immediately during overlapping period when the participants on morning and afternoon shifts were met on the ward. A few were met on night duty on request.

\section{Ethical Considerations}

The research proposal was submitted to the ethical committee of $\mathrm{UCH}$ who granted permission to carry out the study. A copy of the introductory letter was also submitted to the consultant and the matron incharge of AMTH who granted the permission to collect data. Their confidentiality was assured and the fact that names were not required on the questionnaire as well as the possibility to withdraw at any stage of the study was re-emphasized. Contact addresses as well as phone number and e-mail addresses of the investigators were given to the respondents for possibility of further enquiries concerning the study and their participation.

\section{Statistical analysis}

Data were coded and analyzed using the Statistical Package of Social Sciences (SPSS) software, version 15. Frequency tables were used to convey findings of the study and student t-test statistical testing was employed.

\section{Results}

\section{Description of participants}

A total of 385 practicing nurses, who administer injections to patients in their care, from the two hospitals took part in this study. Their average age was 37 years and $7.5 \%$ and $7.7 \%$ of them were men in $\mathrm{UCH}$ and AMTH respectively. With regard to marital status, $42.5 \%$ and $57.7 \%$ of them were married, while
$21 \%$ and $28.9 \%$ were Senior Nursing Officers from $\mathrm{UCH}$ and AMTH respectively.

\section{Knowledge of respondents about Injection Safety}

Respondents' knowledge about injection safety is poor as only $13.2 \%$ and $21.2 \%$ from UCH and AMTH respectively could identify all the points as correct. One third $(33.1 \%)$ of them in UCH identified a safe injection as one in which the waste does not put people at health risk and in AMTH $23.1 \%$ of the participants identified a safe injection as one that will not harm the recipient. There is no significant difference in the knowledge of injection safety among nurses in the two hospitals $(t=0.890)$. This means that the type of hospital does not influence participants' knowledge of injection safety.

\section{Attitude of respondents towards injection safety}

On attitude of respondents towards injection safety: $59 \%$ of the respondents believed that keeping sharps injury records will facilitate education and prevention of injection related injuries, $61 \%$ and $39 \%$ strongly disagreed and disagreed respectively that they boil needles and syringes for re-use. Also 28.3\% and 71.7\% disagreed and strongly disagreed respectively that they autoclave needles and syringes for re-use.

\section{Practice of injection safety}

On practice of injection safety, $7.8 \%$ of injuries sustained by respondents occurred during use, $15.5 \%$ after the use of device and $31.2 \%$ while disposing of the device. Twenty three percent of respondents had sustained injuries from sharps that had been used on HIV positive patients, however only $7.8 \%$ claimed they received post exposure prophylaxis. Furthermore, $76.8 \%$ had never used safety engineered injection devices before and $92.5 \%$ said that such devices are not available in their hospitals. Majority of the respondents (53.2\%) said that nurses are not involved in making policies related to injection safety. Only $30.4 \%$ of respondents reported that their institutions shared sharps injury records regularly, $61.8 \%$ reported that their institutions rarely organize trainings on injection safety, according to $84.4 \%$ of the respondents inserting needles in infusion to remove air is the unsafe injection they identified in their institution. Only $24.4 \%$ frequently leave sharps at patient's bedside. Finally, $79.5 \%$ of them identified doctors as the health 
Table I. t- test table comparing high and low knowledge on practice of injection safety.

\begin{tabular}{llllllll} 
& Knowledge & $\mathrm{N}$ & $\bar{X}$ & SD & T & Df & P \\
\hline \multirow{2}{*}{ Practice of injection safety } & Low & 173 & 54.70 & 3.78 & 9.731 & 383 & $<0.001$ \\
\cline { 2 - 8 } & High & 212 & 59.07 & 4.82 & & & \\
\hline
\end{tabular}

Table II. t- test comparing positive and negative attitude on practice of injection safety

\begin{tabular}{llllllll} 
& Attitude & $\mathrm{N}$ & $\bar{X}$ & $\mathrm{SD}$ & $\mathrm{T}$ & Df & $\mathrm{P}$ \\
\hline \multirow{2}{*}{ Practice of injection safety } & Negative & 158 & 52.59 & 2.468 & 23.780 & \multirow{2}{*}{383} & \multirow{2}{*}{$<0.001$} \\
\cline { 2 - 6 } & Positive & 22 & 60.25 & 3.485 & & & \\
\hline
\end{tabular}

care workers who leave sharps at the patients' bed side. There is a significant difference in the practice of injection safety among nurses in $\mathrm{UCH}$ and those in AMTH ( $\mathrm{t}(398)=4.32, \mathrm{P}<0.05)$. Nurses in $\mathrm{UCH}$ showed higher mean score $(\bar{X}=65.66)$ on practice of injection safety.

There is a significant difference between knowledge and practice of injection safety ( $\mathrm{t}(385)=7.31, \mathrm{p}<0.05)$. In other words, participants with high knowledge of injection safety practiced better than those who have low knowledge. The nurses with high knowledge of injection safety showed higher mean score on practice, than their counterparts with low knowledge (Table I).

There was a significant difference between negative and positive attitude of injection safety and the practice of injection safety (t $(383)=7.80, \mathrm{P}<0.05)$. In other words nurses who have positive attitude to injection safety, practice better than those who have negative attitude. The table has thus revealed that nurses with positive attitude to injection safety showed higher mean score than their counterparts with negative attitude (Table II).

There is no significant difference in participants' grade and their practice of injection safety $(\mathrm{t}(383)=3.45$, $p>0.05)$. Participants of higher grade did not show higher mean score $(\bar{X}=56.70)$ when compared with lower grade nurses $(\bar{X}=57.38)$.

\section{Discussion}

The first research question states: "What is the level of knowledge of nurses about injection safety?" All the respondents claimed they have heard about injection safety before, but $17.8 \%$ of $\mathrm{UCH}$ and $10.6 \%$ of AMTH respondents displayed low knowledge level; claiming that it is giving injection to patients who requested for it. In addition, $63 \%$ of participants from $\mathrm{UCH}$ and $36.5 \%$ from AMTH claimed that double handed recapping is an unsafe injection practice, this agrees with the finding by Hauri et al. that avoiding needle recapping and other hand manipulation is essential to prevent needle stick injuries. ${ }^{2}$ Generally, findings from the current study show that nurses possess high knowledge about injection safety, and this finding contrasts the study of Askarian and Malekmakan that health care personnel including nurses have a background of insufficient knowledge about injection safety. ${ }^{6}$ The high knowledge possessed by respondents in this study could be explained from their being exposed to trainings on injection safety. It is important to note however, that the participants' knowledge at $\mathrm{UCH}$ is not significantly different from their counterparts in AMTH.

The second research question about the participants attitude towards injection safety showed that majority of them (59\%) have positive attitude to injection safety. Nevertheless, of the $67 \%$ who reported no availability of safety engineered injection devices in 
their institutions; only $8.85 \%$ and $24.9 \%$, respectively, strongly agreed that such devices are not affordable to patients. This contradicts the finding of a Korean study by Smith et al. which showed that nurses do not possess positive attitude to injection safety. ${ }^{7}$

"What are the unsafe injection practices that may be targeted for injection safety intervention activities?" is the third research question. The finding shows that the majority $(67.6 \%$ and $40.4 \%)$ of the respondents from $\mathrm{UCH}$ and AMTH respectively had sustained needle stick injuries in the last one year, only $15.3 \%$ and $16.4 \%$ of $\mathrm{UCH}$ and AMTH respondents, respectively, reported their injuries to their institution. In the same vein, only $7.8 \%$ of the $23.6 \%$ respondents who had sustained injury from devices that had been used on HIV patients had post exposure prophylaxis (PEP). This same act of poor reporting was discovered in Askarian and Malekmakan's study in which most needle stick injuries went unreported.' The finding, however, partly disagreed with Smith and Leggat who found that most Needle Stick Injuries (NSI) went unreported because the injuries had occurred when the injection devices had not been used on patients. ${ }^{8}$ This finding is supported by results earlier obtained by Lee et al. ${ }^{9}$ and Center for Disease Prevention and Control (CDC), ${ }^{10}$ which also buttressed the fact that about $30 \%$ of NSIs go unreported in the United States of America while World Health Organization ${ }^{11}$ claimed that NSIs are virtually undocumented in the developing countries. Poor attitude to reporting discovered in this study could have resulted from lack of knowledge about the reporting channels in the hospitals, $54.4 \%$ of them claimed they did not know about any existing reporting channel in their hospitals.

Another unsafe injection practice revealed in this study is leaving loose disposable needles and syringes in various places within the hospital wards/units. Over 50 percent of the participants from both hospitals (60.5\% UCH and $53.9 \%$ AMTH) claimed they never left sharps on patients' bedside, when this thus occurs, most respondents admitted that this is due to negligence of duty, while $30.9 \%$ from both institution claimed that when needles are left on patient's bedside, it is for the purpose of reusing the device on same patient and not others. Nevertheless, it is expected that nurses should know that reuse of injection devices on patient can predispose the patients as well as healthcare providers to risk of blood borne diseases.

The fourth research question: "Do the facilities have necessary requirements for ideal injection safety?" Results showed that majority, (53\%) strongly agreed that conventional needles and syringes are generally used in their hospitals, $67 \%$ of them claimed that injection with safety devices are not available in their hospitals. Only $15.1 \%$ had used safety engineered injection devices before. The above findings show that the hospital management of selected hospitals has not made it a point of duty to provide safety engineered injection devices for use by nurses. This in turn will result in re-use of needles and syringes on patients thereby predisposing the patients as well as the nurses to hazards. This is supported by a study reported in the safe injection manual, "Do No Harm"12 in some health facilities in Nigeria that $45 \%$ of the patients had received injection at least once within the last 12 months in which $12 \%$ of them bought their own syringes and needles for vaccinations, 11 out of 80 facilities were without needles and syringes for more than 3 months, $5 \%$ of providers have reused a disposable needle or syringe on a second patient due to emergency or depleted stock and many of the facilities had no soap and clean water for hand washing. In addition, $43 \%$ of the facilities had no dedicated working table or tray for preparing injections.

On the fifth research question that state "Are there hospital policies about injection safety in the selected setting and what is the involvement of nurses in such policies? In the study, $62.1 \%$ of the respondents reported that such policies are not in existence in their hospitals, $46.8 \%$ claimed that nurses are not involved in making policies about injection safety and only $38.4 \%$ reported that their institutions share sharps injury report regularly. Related finding was demonstrated by Odeyemi et al. in that there were no policies about NSIs. ${ }^{13}$ Lack of such policies might have stemmed from inadequate recording and reporting of sharp injuries among nurses.

\section{Conclusion}

The result of the study shows that the nurses who participated in this study have high knowledge about and positive attitude towards injection safety. 
Nevertheless their practice of injection safety seems to be on the low ebb, due to non-availability of safety engineered injection devices, lack of good reporting mechanism as well as poor availability of policies guiding safe injection practices. Nurses, as health care providers, administer injections to patients which may predispose them (both patient and nurse) to diverse hazards in the process. The patients and the community too may suffer some degree of hazards if injections are not administered in a safe way.

It is therefore, imperative for nurses to utilize the knowledge they have gained from seminars, workshops, conferences, journals and so on, to ensure that injections are administered in a safer manner. Educational programs must be organized to keep empowering nurses on injection safety.

\section{References}

1. Canadian Needles Stick Surveillance Network (CNSSN). Summary of exposures due to percutaneous injuries. 1 April 2000 to 31 March 2001.

2. Hauri AM, Armstrong GL, Hutin Y. Contaminated injections in health care settings in comparative quantification of health risks. Global and regional burden of disease attributable to selected major factors 2003.

3. Hunti $Y$, Hauri A, Chiarello $L$, et al. The members of the injection safety best practices development group. Best infection control practices for intra-dermal, subcutaneous and intra-muscular needle injections. Bull World Health Organ 2003; 81: 491-500.
4. Dicko M, Oni A, Ganivet S, Kone S, Pierre L, Jacquet B. Safety of immunization injections in Africa not simply a problem of logistics. Bull World Health Organ 2000; 76: 163-169.

5. Logez S. Increased access to injection equipment in Burkina Faso: when essential drug programmes improve injection safety. In Safe Injection Global Network (SIGN) 2001. Annual Meeting Report, 30-31.

6. Askarian M, Malekmakan L. The prevalence of needle stick injuries in medical, dental, nursing and midwifery students at the University Hospitals of Shiraz, Iran. Indian I Med Sci 2006; 60(2): 227-232. http://dx.doi.org/10.4103/0019-5359.25904

7. Smith DR, Choe MR, Jeong JS, Jean MY, Chae YR, An GJ. Epidemiology of needle stick and sharps injuries among professional Korean Nurses. J Prof Nur 2006; 22(6): 359-366. http://dx.doi.org/10.1016/j.profnurs.2006.10.003

8. Smith DR, Leggat PA. Needle stick and sharps Injuries among nursing students. World Health Organization 2003. Injection safety: guiding principles to ensure injection device security 2004. Geneva: World Health Organization.

9. Lee JM, Botteman MF, Xanthakos N, Nicklasson L. Needlestick Injuries in the United States. epidemiologic, economic and quality of life issues 2005. Bethesda, MD: AST Associates, Inc.

10. Centre for Disease and Infection Prevention and Control (CDC). Injection safety saves life: reference manual for health workers in Nairobi 2004.

11. World Health Organization. Injection safety. Guiding principles to ensure injection device security. Geneva: World Health Organization 2003

12. Making Medical Injection Safer Team. Do no harm: injection safety in the context of infection prevention and control: trainer's guide. Nigeria: Federal Ministry of Health and John Snow Inc. 2007.

13. Odeyemi KA, Onifade K, Onifade EV. Needle stick/sharp injuries among doctors and nurses at Lagos Universities Teaching Hospital. Nigerian Q J Hosp Med 2005; 15(2): 5054. 